Journal of Animal and Veterinary Advances 11 (4): 561-565, 2012

ISSN: $1680-5593$

(C) Medwell Journals, 2012

\title{
Busulfan Influence the Survival Rate of Chicken Embryos and the Morphology of Gonads
}

\author{
Yang Yu, Gong Ping, Feng Yanping, Yang Yongping, Li Shijun, \\ Peng Xiuli and Gong Yanzhang \\ Key Lab of Agricultural Animal Genetics, Breeding and Reproduction, Ministry of Education, \\ Huazhong Agricultural University, 430070 Wuhan, People's Republic of China
}

\begin{abstract}
Previous studies established the toxicity of busulfan (1, 4-butanediol dimethanesulfonate) suspension in sesame oil or emulsion to Primordial Germ Cells (PGC) and present study examined the survival rate of chicken embryos treated with different dosage of busulfan emulsion $(0,120,240360$ and $480 \mu \mathrm{g})$ then observed the morphology of testes and ovaries at 8.5, 11.5 and 14.5 days. The results showed that low dosage treatment $(<120 \mu \mathrm{g})$ did not have significant influence $(\mathrm{p}>0.05)$ on the survival rate of embryos compared with blank control and medium dosage treatment $(240$ and $360 \mu \mathrm{g})$ significantly reduced the survival rate $(\mathrm{p}<0.05)$, the high dosage treatment $(480 \mu \mathrm{g})$ further reduced the survival rate $(\mathrm{p}<0.05)$. The increasing dosage of busulfan has positive correlation with both the death rate of embryos and the abnormal of the morphology structure in testes and ovaries. About $<120 \mu \mathrm{g}$ busulfan treatment is recommended to obtain recipient chick embryo in producing germline chimera.
\end{abstract}

Key words: Busulfan, testes, ovaries, chicken embryos, toxicity, China

\section{INTRODUCTION}

Eliminating the endogenous $\mathrm{PGC}$ in gonads and then transferring exogenous PGC to the host gonads could produce germline chimera which have potential applications in the generation of transgenic chicken and in research of gonad development. In the past years, soft X-ray irradiation (Li et al., 2001; Naito et al., 2007; Atsumi et al., 2008) surgical operation (Kagami et al., 1997) and chemical agent (Song et al., 2005) were used to remove endogenous $\mathrm{PGC}$ in early developing chicken embryos.

However, these methods have their disadvantages for example, soft X-ray method needs very expensive equipment that many institutes can not afford it surgical operation needs skilled operator. Busulfan, a chemical agent was used as a convenient and suitable tool to eliminate endogenous PGC in many researches (Aige-Gil and Simkiss, 1991; Shin et al., 2008).

Chicken embryonic gonads maintain bipotential from 3.5-6.5 days and then the testes and ovaries begin to differentiate. The medulla of testis is occupied by welldeveloped seminiferous cords and a thin surface epithelium is surrounded. The left ovary is comprised of thick cortex and underlying medulla and the right ovary will degenerate and become vestigial (Smith et al., 2003,
2009; Guioli and Lovell-Badge, 2007). Since, the structures of male and female gonads are different eventually it is not clear whether the sensitivity of the testes and ovary to the treatment of busulfan are the same or not. Previous studies focused on the effect of busulfan suspension or emulsion for the deprivation of endogenous PGC in gonads but didn't consider much about both the survival rate of embryos and the morphology changes of testes and ovaries (Song et al., 2005; Nakamura et al., 2009). The purpose of present research is to study the effects of busulfan on gonads of different sexes. To investigate the effect of busulfan on embryonic embryos, different dosages of busulfan emulsion were used in the early developing chicken embryos then the morphological and structural change of gonads were studied by Haematoxylin and Eosin (HE) staining.

\section{MATERIALS AND METHODS}

A total 540 fertilized white leghorn chicken eggs were obtained from experimental chicken farm, Department of Animal Science of Huazhong Agricultural University. The eggs were randomly divided into six groups and incubated at $37.8^{\circ} \mathrm{C}$ in a humid incubator. Five treated groups were injected a dosages of $0,120,240,360$ and $480 \mu \mathrm{g}$ busulfan, respectively by using the method

Corresponding Author: Gong Yanzhang, Key Lab of Agricultural Animal Genetics, Breeding and Reproduction, Ministry of Education, Huazhong Agricultural University, 430070 Wuhan, People's Republic of China 
described previously by Song et al. (2005). The control group was normal incubating eggs. After injection, the eggs were incubated continuously and 30 eggs were collected, respectively at $8.5,11.5$ and 14.5 days the survival rate of embryos was analyzed using t-test at each stage.

Immunohistochemical identification of gonocytes in gonads: The urogenital systems were rapidly removed and fixed immediately in 4\% Paraformaldehyde (PFA) overnight. All samples were routinely dehydrated in an ethanol series from low to high and subsequently embedded in paraffin. The samples were then dissected into sections of $6 \mu \mathrm{m}$ thickness and mounted on poly-1lysine-coated glass slides. All the slides were dried overnight at $37^{\circ} \mathrm{C}$. Streptavidin-Biotin-peroxidase Complex (SABC) Immunohistochemical method was proceeded as described previously (Zhang et al., 2009) by using the antibody of SSEA-1 (Santa cruz).

Haematoxylin and eosin staining: To understand the morphological and structural changes of testes treated by busulfan of different dosage, haematoxylin and eosin staining were used on the adjacent sections by using the method described by Zhang et al. (2009).

\section{RESULTS AND DISCUSSION}

Survival rate of embryos: Embryos treated with busulfan showed no developmental delaying but presented some abnormalities including small eyes or died when hatching. The survival rates of embryos treated with different dosages of busulfan evaluated, respectively at days 8.5 , 11.5 and 14.5 after setting were shown in Table 1 . It showed from the table that there was no significant difference in survival rate at $8.5,11.5$ and 14.5 days, respectively among the blank control (normal embryos), negative control (injection of emulsion only) and low dosage group (injection of $120 \mu \mathrm{g}$ busulfan) ( $\mathrm{p}>0.05$ ).

Table 1: Survival rate of embryos after injection of different dosage of busulfan treatment

\begin{tabular}{lccc}
\hline & Percentage surviving $($ days $)$ & \\
Busulfan dose & 8.5 & 11.5 & 14.5 \\
\hline Blank control $(\mu \mathrm{g})$ & $83.3(\mathrm{n}=30)^{\mathrm{a}}$ & $82.8(\mathrm{n}=29)^{\mathrm{a}}$ & $82.1(\mathrm{n}=28)^{\mathrm{a}}$ \\
0 & $83.3(\mathrm{n}=30)^{\mathrm{a}}$ & $81.5(\mathrm{n}=27)^{\mathrm{a}}$ & $78.6(\mathrm{n}=28)^{\mathrm{a}}$ \\
120 & $81.5(\mathrm{n}=27)^{\mathrm{a}}$ & $76.7(\mathrm{n}=30)^{\mathrm{a}}$ & $76.0(\mathrm{n}=25)^{\mathrm{a}}$ \\
240 & $60.0(\mathrm{n}=30)^{\mathrm{b}}$ & $46.2(\mathrm{n}=26)^{\mathrm{b}}$ & $50.0(\mathrm{n}=24)^{\mathrm{b}}$ \\
360 & $64.0(\mathrm{n}=25)^{\mathrm{b}}$ & $47.8(\mathrm{n}=23)^{\mathrm{b}}$ & $45.8(\mathrm{n}=24)^{\mathrm{b}}$ \\
480 & $37.5(\mathrm{n}=24)^{\mathrm{c}}$ & $22.2(\mathrm{n}=27)^{\mathrm{c}}$ & $21.4(\mathrm{n}=28)^{\mathrm{c}}$ \\
\hline Means with different superscripts in the same column are significantly \\
different $(\mathrm{p}<0.05)$
\end{tabular}

However, The survival rates of embryos treated with 240,360 and $480 \mu \mathrm{g}$ busulfan were all significantly lower than the controls $(p<0.05)$. As, the dosage of the busulfan increased the mortality rate of embryos increased anyhow, there was no significant difference between the treatment with dosage of 240 and $360 \mu \mathrm{g}(\mathrm{p}>0.05)$. It seemed that the higher the dosage of busulfan injected, the less the embryos surviving at the late developing period but the precentage of surviving decreasing in a stepwise way.

Busulfan reduces the PGCs in gonads: IHC was proceeded to identify SSEA-1 positive gonocytes in chicken gonads with $120 \mu \mathrm{g}$ busulfan treatment at 5.5 days (Fig. 1). The results showed that busulfan can effectively reduce the PGCs in chicken embryo testes and ovaries.

Structural and morphological changes of gonads by $\mathrm{HE}$ staining: $\mathrm{HE}$ staining was performed to compare the morphological and structural changes of embryos treated with different dosages of busulfan. In the controls, well-developed seminiferous cords occupied the medulla and a thin surface epithelium could be observed in left and right testes and the gonocytes spread within the medulla (Fig. 2A-C). When treated with busulfan at the low dosage $(120 \mu \mathrm{g})$, the organization and structure of the testes was not affected until 14.5 days but at medium and high dosage $(240,360$ and $480 \mu \mathrm{g})$, the normal organization and structure was damaged the seminiferous cords became fewer and poorly organized and the interstitium unusually expanded (Fig. 2A4, B3, B4, C2, C3, C4). It could be observed that the time of damage in the

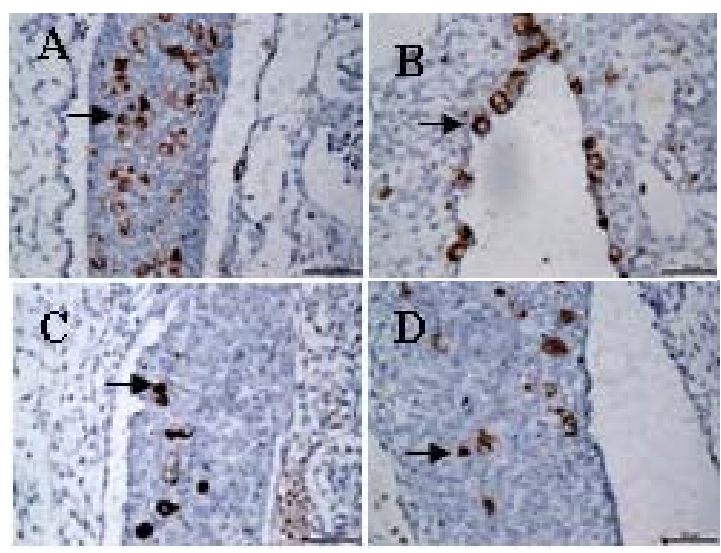

Fig. 1: SSEA-1 positive PGCs in the embryonic gonads at 5.5 days. A, B: Control male and female embryos. C, D: Male and female embryos injected with $120 \mu \mathrm{g}$ busulfan suspended in $80 \mu \mathrm{L}$ mineral oil, respectively. It showed positive $\mathrm{PGCs}$ were much fewer in treated embryos than control embryos. About 10 ocular $\mathrm{x} 40$ objective 


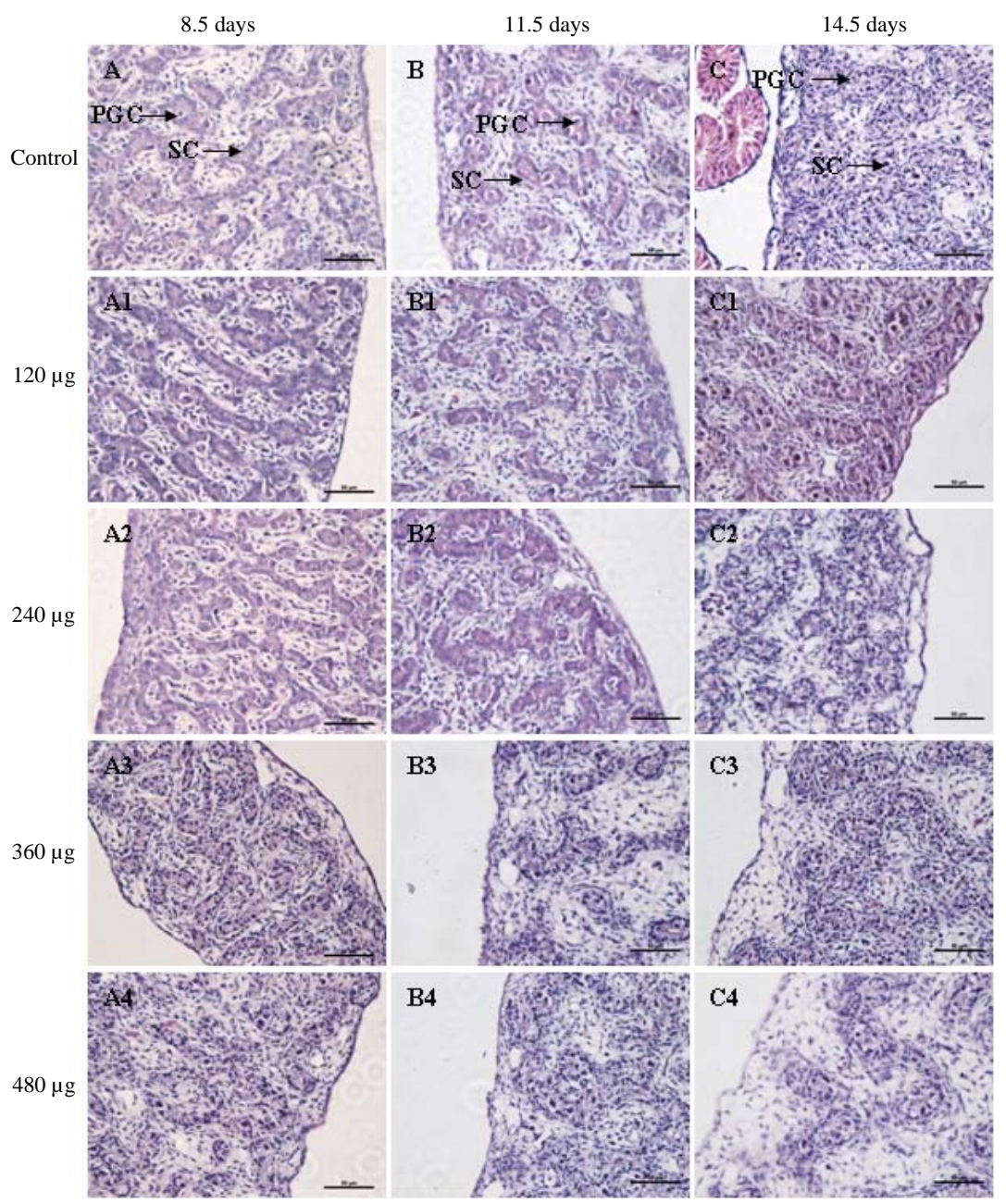

Fig. 2: Micrographs of testes treated with $0 \mu \mathrm{g}$ (A-C), $120 \mu \mathrm{g}$ (A1-C1), $240 \mu \mathrm{g}$ (A2-C2), $360 \mu \mathrm{g}$ (A3-C3) and $480 \mu \mathrm{g}$ (A4-C4) busulfan emulsion. A-A4: at 8.5 days; B-B4: at 11.5 days; C-C4: at 14.5 days. Busulfan reduces the number of Seminiferous Cords (SC) at 11.5 and 14.5 days. PGC represents primary germ cell (10 ocular $\mathrm{x} 40$ objective). HE staining

organization and structure in testes was related the dosage used with the higher the dosage used the earlier the damage began. For instance at a dosage of $480 \mu \mathrm{g}$, the damage began at 8.5 days and lasted to 14.5 days and at $360 \mu \mathrm{g}$, it began at 11.5 days and also there was damage at 14.5 days but at $240 \mu \mathrm{g}$ it began late at 14.5 days. It was unclear if there was any damage of the organization and the structure in the testes after 14.5 days at the low dosage $\left(\begin{array}{ll}120 & \mu \mathrm{g}\end{array}\right)$. Control ovary showed typical asymmetric development that was characterized by a large left ovary and a smaller regressing right ovary. At the histological level, the left ovary had a well developed outer cortex and a vacuolated medulla riddled with lacunae (Fig. 3A-C). The ovaries at 8.5 and 11.5 days showed normal medulla and thinner cortex when treated with different dosages of busulfan emulsion (Fig. 3A3, A4, B3 and B4). The embryos treated with 360 and $480 \mu \mathrm{g}$ busulfan caused a reduction in the thickness of the cortex and the development of lacunae at 14.5 days (Fig. 3C and C4). It is found that gonads have response to busulfan treatment and the IHC results and the changes of the histological structure of gonads have confirmed it. Song et al. (2005) showed that $75 \mu \mathrm{g}$ busulfan emulsion resulted in $38 \%$ survival rate at the 6th embryonic day. The survival rate was significantly lower than in the control group.

However, recent research obtained over $80 \%$ survival rate in blank control group and about $70 \%$ survival rate in treated group at the 7th embryonic day (Nakamura et al., 2009), this result was similar to present study. The 

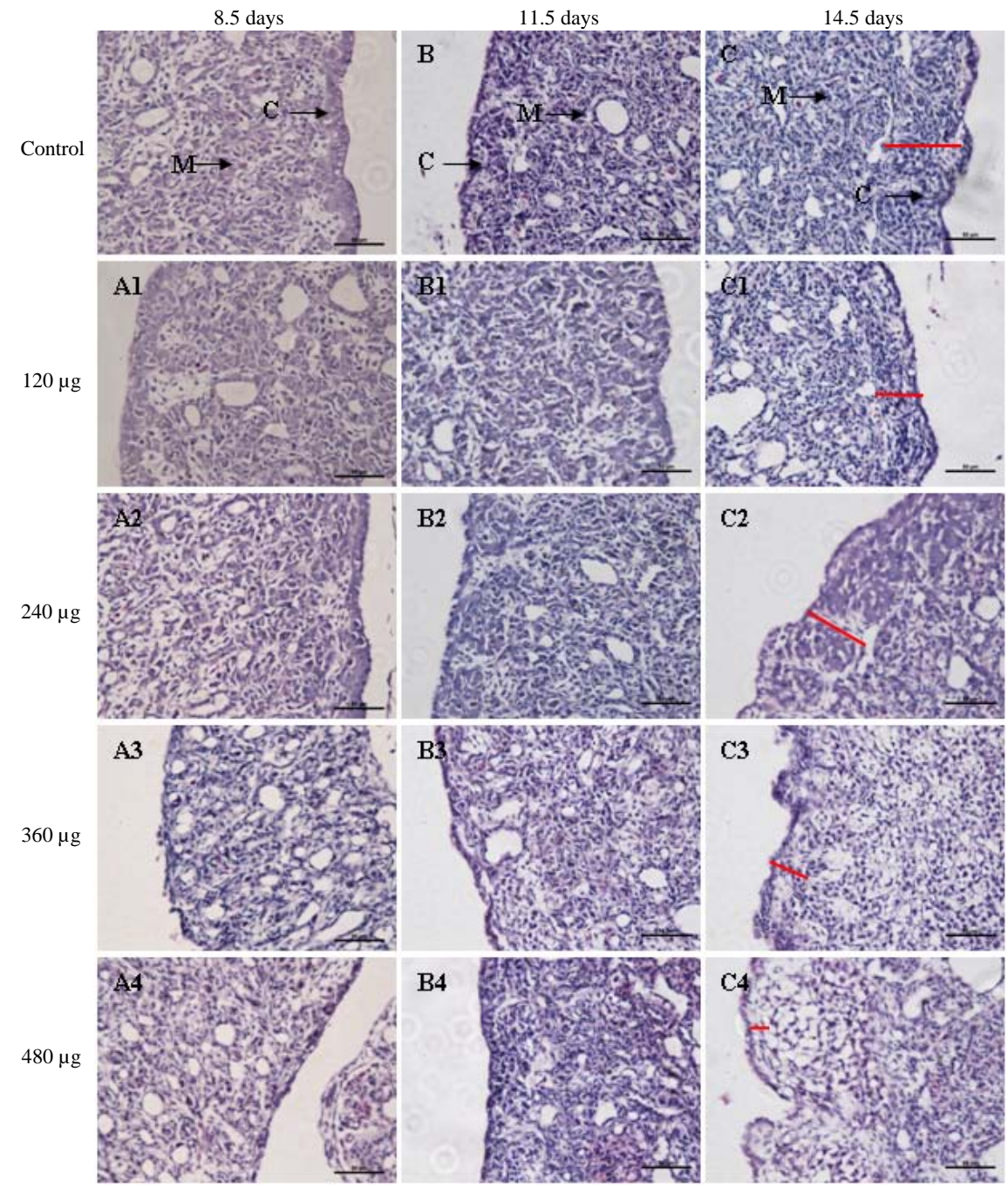

B4

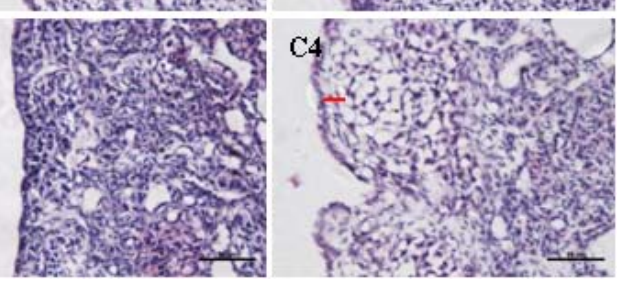

Fig. 3: Micrographs of left ovaries treated with $0 \mu \mathrm{g}$ (A-C), $120 \mu \mathrm{g}$ (A1-C1), $240 \mu \mathrm{g}$ (A2-C2), $360 \mu \mathrm{g}$ (A3-C3) and $480 \mu \mathrm{g}$ (A4-C4) busulfan emulsion. A-A4: at 8.5 days; B-B4: at 11.5 days; C-C4: at 14.5 days. Busulfan lower the thickness of Cortex (C) at 14.5 days. The gonads treated with 360 and $480 \mu \mathrm{g}$ busulfan showed abnormal morphology at 14.5 days. The red bar shows the thickness of cortex. M represents medulla (10 ocular x40 objective). HE staining

survival rate of chicken embryo was decreased stepwise with the increasing of busulfan treatment. In the research it is found when treated with low dosage of busulfan $(120 \mu \mathrm{g})$, morphology and structure of embryonic testes are normal but when treated with $240 \mu \mathrm{g}$ or more busulfan they are damaged.

It seems that the dosage of busulfan should be above a threshold when it will impact the morphology and structure of embryonic testes. Vick et al. (1993) found that injection of $250 \mu \mathrm{g}$ busulfan reduced the number of testicular cords and this is consistent with the result. Song et al. (2005) confirmed that busulfan directly depletes PGC by degenerating PGC rather than the somatic cells of the gonads. They pointed out that busulfan selectively cytotoxic to PGC at low dosages. It is presumed that busulfan have accumulative cytotoxic to gonads and embryos at medium and high dosages which results in the abnormal gonads structure and the high death rate.

Busulfan influencing the gonocytes and the histological structure of gonad will result in the death of embryos finally.

\section{CONCLUSION}

Testes have different response to diversity busulfan treatment. About $<120 \mu \mathrm{g}$ busulfan treatment could reduce the PGCs but did not effect on the survival rate of 
embryos and the morphology structure of chicken embryonic gonads. Therefore, the dosage of busulfan treatment is suggested to $<120 \mu \mathrm{g}$ in transgenic chicken.

\section{ACKNOWLEDGEMENTS}

The researchers thank G-Y Zhang, the staff of Wuhan Guge Biotechnique Company for technical assistance and proof reading. This research was supported by National Natural Science Foundation of China (project No. 30671494 and 30800781) and New faculty funding of Ministry of Education of P.R. China (200805041054).

\section{REFERENCES}

Aige-Gil, V. and K. Simkiss, 1991. Sterilisation of avian embryos with busulphan. Res. Vet. Sci., 50: 139-144.

Atsumi, Y., T. Tagami, H. Kagami and T. Ono, 2008. Restriction of germline proliferation by soft X-ray irradiation of chicken embryos and its application to chimera production. J. Poult. Sci., 45: 292-297.

Guioli, S. and R. Lovell-Badge, 2007. PITX2 controls asymmetric gonadal development in both sexes of the chick and can rescue the degeneration of the right ovary. Development, 134: 4199-4208.

Kagami, H., T. Tagami, Y. Matsubara, T. Harumi and H. Hanada et al., 1997. The developmental origin of primordial germ cells and the transmission of the donor-derived gametes in mixed-sex germline chimeras to the offspring in the chicken. Mol. Reprod. Dev., 48: 501-510.

Li, H.C., H. Kagami, K. Matsui and T. Ono, 2001. Restriction of proliferation of primordial germ cells by the irradiation of Japanese quail embryos with soft X-rays. Comp. Biochem. Physiol. Mol. Integr. Pshysiol., 130: 133-140.
Naito, M., T. Harumi, T. Minematsu, A. Tajima and T. Kuwana, 2007. Effect of soft X-ray irradiation on the migratory ability of primordial germ cells in chickens. Br. Poult. Sci., 48: 121-126.

Nakamura, Y., F. Usui, Y. Atsumi, A. Otomo and A. Teshima et al., 2009. Effects of busulfan Sustained-release emulsion on depletion and repopulation of primordial germ cells in early chicken embryos. J. Poult. Sci., 46: 127-135.

Shin, S.S., T.M. Kim, S.Y. Kim, T.W. Kim and H.W. Seo et al., 2008. Generation of transgenic quail through germ cell-mediated germline transmission. FASEB J., 22: 2435-2444.

Smith, C.A., K.N. Roeszler, T. Ohnesorg, D.M. Cummins, P.G. Farlie, T.J. Doran and A.H. Sinclair, 2009. The avian Z-linked gene DMRT1 is required for male sex determination in the chicken. Nature, 461: 267-271.

Smith, C.A., M. Katz and A.H. Sinclair, 2003. DMRT1 is upregulated in the gonads during female-to-male sex reversal in ZW chicken embryos. Biol. Reprod., 68: $560-570$.

Song, Y., S. D'Costa, S.L. Pardue and J.N. Petitte, 2005. Production of germline chimeric chickens following the administration of a busulfan emulsion. Mol. Reprod. Dev., 70: 438-444.

Vick, L., G. Luke and K. Simkiss, 1993. Germ-line chimaeras can produce both strains of fowl with high efficiency after partial sterilization. J. Reprod. Fertil., 98: 637-641.

Zhang, G.Y., Y. Wang, K.M. Peng, C. Wang, G. Tian and H.Z. Liu, 2009. Strategic Localization of Toll-like receptor 4 in the digestive tract of blunt snout bream (Megalobrama amblycephala). Anat. Histol. Embryol., 38: 401-405. 